\title{
Assessment of the anthelmintic activity of medicinal plant extracts and purified condensed tannins against free-living and parasitic stages of Oesophagostomum dentatum
}

\author{
Andrew R Williams ${ }^{1 *}$, Honorata M Ropiak², Christos Fryganas², Olivier Desrues ${ }^{1}$, Irene Mueller-Harvey²
} and Stig M Thamsborg ${ }^{1}$

\begin{abstract}
Background: Plant-derived condensed tannins (CT) show promise as a complementary option to treat gastrointestinal helminth infections, thus reducing reliance on synthetic anthelmintic drugs. Most studies on the anthelmintic effects of CT have been conducted on parasites of ruminant livestock. Oesophagostomum dentatum is an economically important parasite of pigs, as well as serving as a useful laboratory model of helminth parasites due to the ability to culture it in vitro for long periods through several life-cycle stages. Here, we investigated the anthelmintic effects of $\mathrm{CT}$ on multiple life cycle stages of $O$. dentatum.

Methods: Extracts and purified fractions were prepared from five plants containing CT and analysed by HPLC-MS. Anthelmintic activity was assessed at five different stages of the $O$. dentatum life cycle; the development of eggs to infective third-stage larvae ( $L 3)$, the parasitic $L 3$ stage, the moult from $L 3$ to fourth-stage larvae ( $L 4)$, the $L 4$ stage and the adult stage.

Results: Free-living larvae of $O$. dentatum were highly susceptible to all five plant extracts. In contrast, only two of the five extracts had activity against $L 3$, as evidenced by migration inhibition assays, whilst three of the five extracts inhibited the moulting of $L 3$ to $L 4$. All five extracts reduced the motility of $L 4$, and the motility of adult worms exposed to a CT-rich extract derived from hazelnut skins was strongly inhibited, with electron microscopy demonstrating direct damage to the worm cuticle and hypodermis. Purified $C T$ fractions retained anthelmintic activity, and depletion of CT from extracts by pre-incubation in polyvinylpolypyrrolidone removed anthelmintic effects, strongly suggesting $C T$ as the active molecules.

Conclusions: These results suggest that CT may have promise as an alternative parasite control option for $O$. dentatum in pigs, particularly against adult stages. Moreover, our results demonstrate a varied susceptibility of different life-cycle stages of the same parasite to $\mathrm{CT}$, which may offer an insight into the anthelmintic mechanisms of these commonly found plant compounds.
\end{abstract}

Keywords: Oesophagostomum dentatum, Plant extracts, Condensed tannins, Anthelmintic

\footnotetext{
*Correspondence: arw@sund.ku.dk

${ }^{1}$ Department of Veterinary Disease Biology, Faculty of Health and Medical

Sciences, University of Copenhagen, Frederiksberg, Denmark

Full list of author information is available at the end of the article
} 


\section{Background}

Parasitic worms (helminths) of the gastrointestinal (GI) tract are pathogens of major global importance. Over a billion people, mainly in developing countries, are estimated to be infected with soil-transmitted helminths, whilst helminth infection is also a serious problem in livestock production worldwide, causing significant economic losses and threatening food security [1-3]. Control of helminths relies almost exclusively on a limited number of synthetic anthelmintic drugs. The limitations of this reliance on chemotherapy are the threat of parasites developing resistance to drug treatment (already widespread in some livestock production systems) $[4,5]$, the cost of drugs for small-scale farmers in developing countries and for some helminths, lack of efficacy of current available drugs [1]. Therefore, novel and complementary helminth control options are urgently needed.

The use of natural plant extracts as de-wormers for humans and livestock has long been practiced, however scientific validation of these practices and identification of active compounds has been lacking [6-8]. Anthelmintic effects of plants are normally ascribed to secondary metabolites such as alkaloids, terpenoids or polyphenols such as proanthocyanidins [9], also known as condensed tannins (CT). Proanthocyanidins are a diverse and widely-occurring group of compounds, and consist of polymers of either catechin and/or epicatechin (termed procyanidins - PC), or of gallocatechin and/or epigallocatechin (termed prodelphinidins - PD), with hetero-polymers being common [10]. They are found in plant material from both tropical and temperate areas, and have been widely investigated for their antioxidant and anti-inflammatory properties $[11,12]$. It is also apparent that CT can have anthelmintic effects; reduced worm burdens have been reported in rats administered CT in the diet, or in livestock grazing forages containing CT $[13,14]$. Moreover, direct anthelmintic effects of purified CT have been confirmed in in vitro assays against, amongst others, Haemonchus contortus [15], Ostertagia ostertagi [16] and Ascaris suum [17]. However, much work remains to be done to establish the spectra of activity of CT, i.e. the range of helminth species that are susceptible, and what stages of the life cycle are targeted by these molecules.

Oesophagostomum dentatum is a common helminth parasite that resides in the large intestine of pigs, and is of economic importance as it causes significant production losses for farmers [18-20]. Resistance of O. dentatum to levamisole, pyrantel and possibly ivermectin has been reported in Europe [21,22]. Moreover, O. dentatum also serves as a good model parasite due to its amenability to culture in the laboratory - several different lifecycle stages can be easily maintained and manipulated, and hence this worm is being increasingly used as a model parasite for biological investigations [23]. In the present study we investigated 1) whether CT have direct anthelmintic activity against $O$. dentatum in vitro, and 2 ) which stages of the life cycle of this parasite were specifically targeted by CT.

\section{Methods \\ Extraction of plant material and analysis of proanthocyanidins}

Five sources of plant material were selected, on the basis of known high concentrations of CT and/or use as traditional medicinal plants. Three traditional medicinal plants were obtained from Flos (Mokrsko, Poland) these were leaves from blackcurrant (Ribes nigrum), flowers from Tilia (Tilia L., a mixture of T. cordata, T. platyphyllos and T. vulgaris) and bark from willow (Salix spp.). Flowers from white clover (Trifolium repens) were obtained from Ziola Kurpi (Jednorozec, Poland). Extraction and analysis were as previously described [17]. Briefly, plant material was extracted with acetone/water (excluding addition of ascorbic acid). To purify CT, extracts were applied to Sephadex LH-20 columns, and eluted with acetone/water (3:7 and 1:1, v/v). Condensed tannin content was quantified by thiolytic degradation [16] and HPLC [17] with the use of dihydroquercetin as external standard. The identification of compounds was confirmed by LC-MS [17]. In addition, skins from hazelnut (Corylus avellana), which had previously been shown to contain CT with potent in vitro anthelmintic effects against $A$. suum [17], were prepared as previously described.

\section{Larval development assay with free-living stages}

All animal experimentation was conducted under the guidelines and with approval of the Danish Animal Experimentation Inspectorate (Licence number 2010/561-1914). Two 8-week-old pigs were each infected with two doses of 5,000 third-stage larvae (L3) O. dentatum by stomach tube, three weeks apart. Upon patency, eggs were isolated from freshly collected faeces by sieving and saturated salt flotation [24].

For the development assay, plant extracts were dissolved in distilled water at appropriate concentrations and added to 96 -well plates together with $15 \%(\mathrm{v} / \mathrm{v})$ of a larval feeding solution (1\% yeast extract (Sigma-Aldrich) in Earle's balanced salt solution), antibiotics $(300 \mathrm{U} / \mathrm{mL}$ penicillin, $300 \mu \mathrm{g} \mathrm{mL} /$ streptomycin $)$ and antimycotic $(10 \mu \mathrm{g} / \mathrm{mL}$ amphotericin B). Negative and positive controls consisted of water and $50 \mu \mathrm{g} / \mathrm{mL}$ levamisole, respectively. One hundred eggs were then added per well and the plates incubated at $25^{\circ} \mathrm{C}$ in a humidified environment for 7 days. Larvae were then killed by the addition of iodine and the numbers of L3 per well were determined.

\section{Larval migration assay}

Third-stage larvae were produced from the faeces of mono-infected donor pigs by standard copro-culture, 
collected by Baermann apparatus and stored in water at $10^{\circ} \mathrm{C}$. On the day of the assay, L3 were rapidly exsheathed by the addition of $2 \%$ sodium hypochlorite (10-15\% available chloride, Sigma-Aldrich). L3 were then washed five times in sterile water. To assess the migratory ability of the L3, we performed a migration inhibition assay essentially as described previously [17]. Briefly, larvae were suspended in RPMI 1640 media with HEPES (Gibco) containing L-glutamine (2 mM), $100 \mathrm{U} /$ $\mathrm{mL}$ penicillin and $100 \mu \mathrm{g} / \mathrm{mL}$ streptomycin. One hundred larvae were then added in triplicate to each well of a 48-well plate containing medium with either plant extracts or purified CT fractions, $50 \mu \mathrm{g} / \mathrm{mL}$ ivermectin (positive control) or medium alone (negative control). The larvae were then incubated overnight at $37^{\circ} \mathrm{C}$ in an atmosphere of $5 \% \mathrm{CO}_{2}$, before addition of agar to a final concentration of $0.8 \%$. After a four hour migration period, the numbers of larvae able to migrate out of the setting agar were counted by light microscopy. In some experiments, plant extracts were pre-incubated with polyvinylpolypyrrolidone (PVPP) to selectively deplete $\mathrm{CT}$ - this was done by overnight incubation as described previously [17].

\section{Larval development assay with parasitic stages}

Third-stage larvae were exsheathed as above, washed and then 100 larvae per well were seeded in $2 \mathrm{~mL}$ of culture medium (LB broth containing 10\% heat-inactivated porcine serum, $200 \mathrm{U} / \mathrm{mL}$ penicillin, $200 \mu \mathrm{g} / \mathrm{mL}$ streptomycin and $1 \mu \mathrm{g} / \mathrm{mL}$ amphotericin B) in 24-well plates. Acetone/water plant extracts were added at a concentration of $1 \mathrm{mg} / \mathrm{mL}$. Positive control wells received $25 \mathrm{mM}$ of diethylcarbamazine (Sigma-Aldrich) in order to block the development from third-stage to fourth-stage larvae, whilst negative control wells received only culture medium. The larvae were then incubated at $37^{\circ} \mathrm{C}$ in an atmosphere of $5 \% \mathrm{CO}_{2}$ for 14 days, with the medium being replenished on days 5 and 10. On day 14 the percentage of L4 in each well was determined.

\section{Motility assay with fourth-stage larvae}

Third-stage larvae were exsheathed as above, washed and then seeded at a concentration of 1000 larvae/mL in tissue culture flasks containing complete LB broth culture medium. Larvae were then incubated at $37^{\circ} \mathrm{C}$ in an atmosphere of $5 \% \mathrm{CO}_{2}$ with medium changes every five days. Larvae were cultured for 21-24 days to allow development of large numbers of L4. The L4 were then separated from L3 by repeated sedimentation, washed five times in warm sterile saline, and then suspended in the same medium as used for the L3 migration assay above. Approximately 10 larvae were then added to each well of a 48-well plate containing either acetone/ water plant extracts or purified CT fractions, medium only or ivermectin $(50 \mu \mathrm{g} / \mathrm{mL})$. The motility of the larvae was then scored daily for four days. Motility was scored on a $0-5$ scale where 0 is no movement and 5 is vigorous movement, as described in more detail by Stepek et al. [25].

\section{Motility assay with adult worms}

Donor pigs were infected with $O$. dentatum larvae as described above. Approximately five weeks after the second infective dose, the pigs were killed by captive bolt pistol and exsanguination, and the large intestine was removed. Adult worms were manually plucked from the gut contents with forceps and washed well with warm saline. The worms were then taken to the laboratory and washed repeatedly in the same sterile culture medium as used for the L3 migration assay. Four worms were then seeded in each well of a 24-well plate containing either medium only, ivermectin $(50 \mu \mathrm{g} / \mathrm{mL})$ or hazelnut skin extract. The worms were incubated at $37^{\circ} \mathrm{C}$ in an atmosphere of $5 \% \mathrm{CO}_{2}$ for three days, with motility assessed twice daily as described above. After 24 hours, a subset of worms from the negative control wells and from the wells containing the highest concentration of CT were washed, fixed and examined by transmission electron microscopy as previously described [17].

\section{Statistical analysis}

The effect of plant extracts and CT fractions on larval migration and moulting was assessed by two-way ANOVA with Bonferonni post-hoc testing. Graphpad Prism 6 was used for the analyses.

\section{Results}

\section{Analysis of plant extracts}

Acetone/water extraction of the five plant materials yielded extracts with varying yields of CT ranging from $13.1 \mathrm{~g} \mathrm{CT} /$ $100 \mathrm{~g}$ extract (white clover flowers) to $73.8 \mathrm{~g} \mathrm{CT} / 100 \mathrm{~g}$ extract for hazelnut skins (Table 1). Fractionation on Sephadex LH-20 with acetone/water yielded a first fraction (F1) containing from 13.3 to $55.4 \mathrm{~g} \mathrm{CT} / 100 \mathrm{~g}$ fraction, whilst the second fraction (F2) contained more CT (ranging from 70.3-96.7 g CT/100 g fraction - Table 1).

\section{Tannin-containing extracts inhibit the development of free-living larvae}

Acetone/water extracts were first tested in the widely-used assay of free-living larval development, which measures the ability of newly-hatched larvae from eggs to develop to infective L3 in the presence of a putative anthelmintic agent. In negative control wells, $80 \%$ of larvae developed to L3 after 7 days. The addition of $50 \mu \mathrm{g} / \mathrm{mL}$ levamisole resulted in $100 \%$ inhibition of egg hatching and therefore subsequent larval development. Egg hatching was not inhibited by the plant extracts, but all five plant extracts 
Table 1 Chemical analysis of plant extracts and derived fractions

\begin{tabular}{ll}
\hline Sample & PAC (g/100 g extract or fraction) \\
\hline Hazelnut skin extract & 73.8 \\
F1 & 51.3 \\
F2 & 70.3 \\
White clover flower extract & 13.1 \\
F1 & 13.3 \\
F2 & 81.9 \\
Blackcurrant leaves extract & 20.2 \\
F1 & 55.4 \\
F2 & 76.8 \\
Willow bark extract & 16.2 \\
F1 & 24.2 \\
F2 & 87.0 \\
Tilia flowers extract & 21.0 \\
F1 & 49.7 \\
F2 & 96.1 \\
\hline 'PAC' - proanthocyanidins, 'F1' - fraction 1, 'F2' - fraction 2. Results from the
\end{tabular}

hazelnut skin extract and fractions were also reported previously in [17].

strongly inhibited the development of L1 to infective L3, with most larvae dying at the L1 or L2 stage (Figure 1). A dose-dependent relationship was evident for all extracts except for hazelnut skin, where all four tested concentrations $(125-1000 \mu \mathrm{g} / \mathrm{mL})$ inhibited development by more than $90 \%$ (Figure 1). Overall, these results clearly show that CT-containing plant extracts have anthelmintic activity against the free-living stages of $O$. dentatum.
Activity of extracts and tannin fractions against parasitic third-stage larvae

Having established that all five extracts had activity against free-living larvae, anthelmintic effects were next assessed against the L3 larval stage that is infective to pigs and develops within the GI tract. First, the migratory ability of exsheathed L3 was quantified after incubation in the extracts. Migration of L3 was reduced $(\mathrm{P}<$ 0.01 ) after overnight incubation with extracts of willow bark and Tilia flowers at a concentration of $1 \mathrm{mg} / \mathrm{mL}$; in contrast, migration was not significantly reduced after incubation in the other three extracts (Figure 2A). To assess whether CT were the active molecules responsible for the inhibitory activity of willow bark and Tilia extracts, the samples were pre-incubated with PVPP, which selectively binds and precipitates CT [10]. Incubation of larvae in these CT-depleted extracts did not affect migratory ability, strongly suggesting $\mathrm{CT}$ as the active compounds (Figure 2B).

To further confirm the role of CT in the observed effects, F1 and F2 fractions were isolated from the acetone/water extracts of willow bark and Tilia and used in migration inhibition assays. The activity of hazelnut skin fractions, which had previously demonstrated potent anthelmintic effects against $A$. suum [17], were also examined. No anthelmintic activity was evident with the F1 fractions at concentrations up to $500 \mu \mathrm{g} / \mathrm{mL}$ of CT (data not shown). However, F2 fractions inhibited migration in a dose-dependent manner (Figure 2C).

We next investigated whether long-term incubation of L3 in the extracts would reduce subsequent moulting to the L4 larval stage. After 14 days incubation in medium

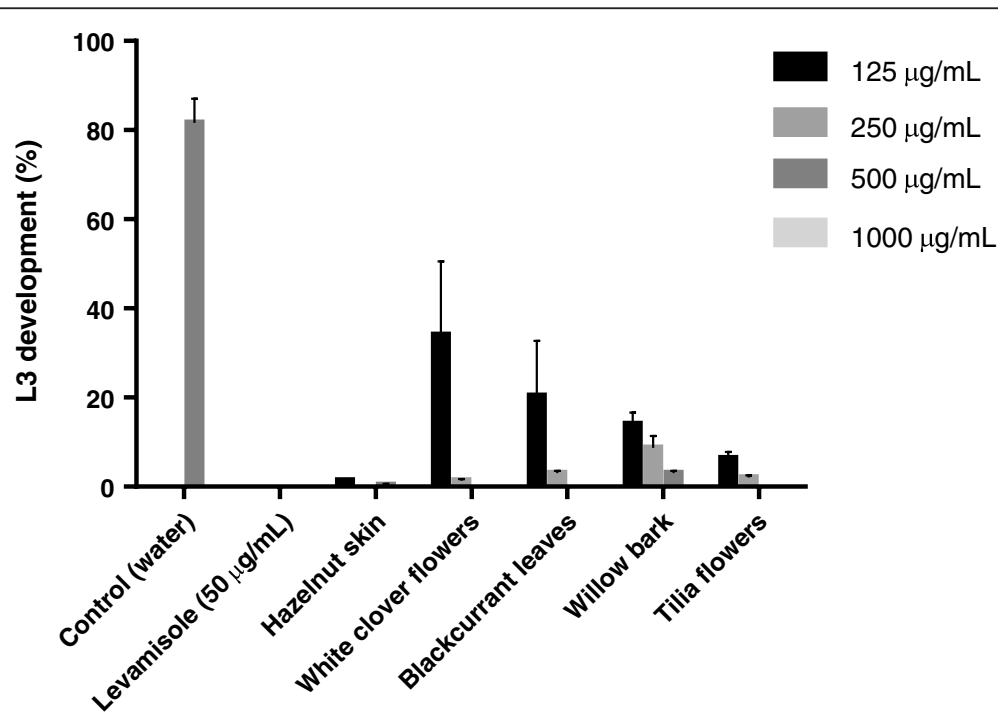

Figure 1 Inhibition of development of Oesophagostomum dentatum larvae by plant extracts. Percentage development of $O$. dentatum free living stages, from eggs through L1-L2 to L3, in the presence of water, levamisole or different concentrations from five plant extracts. 


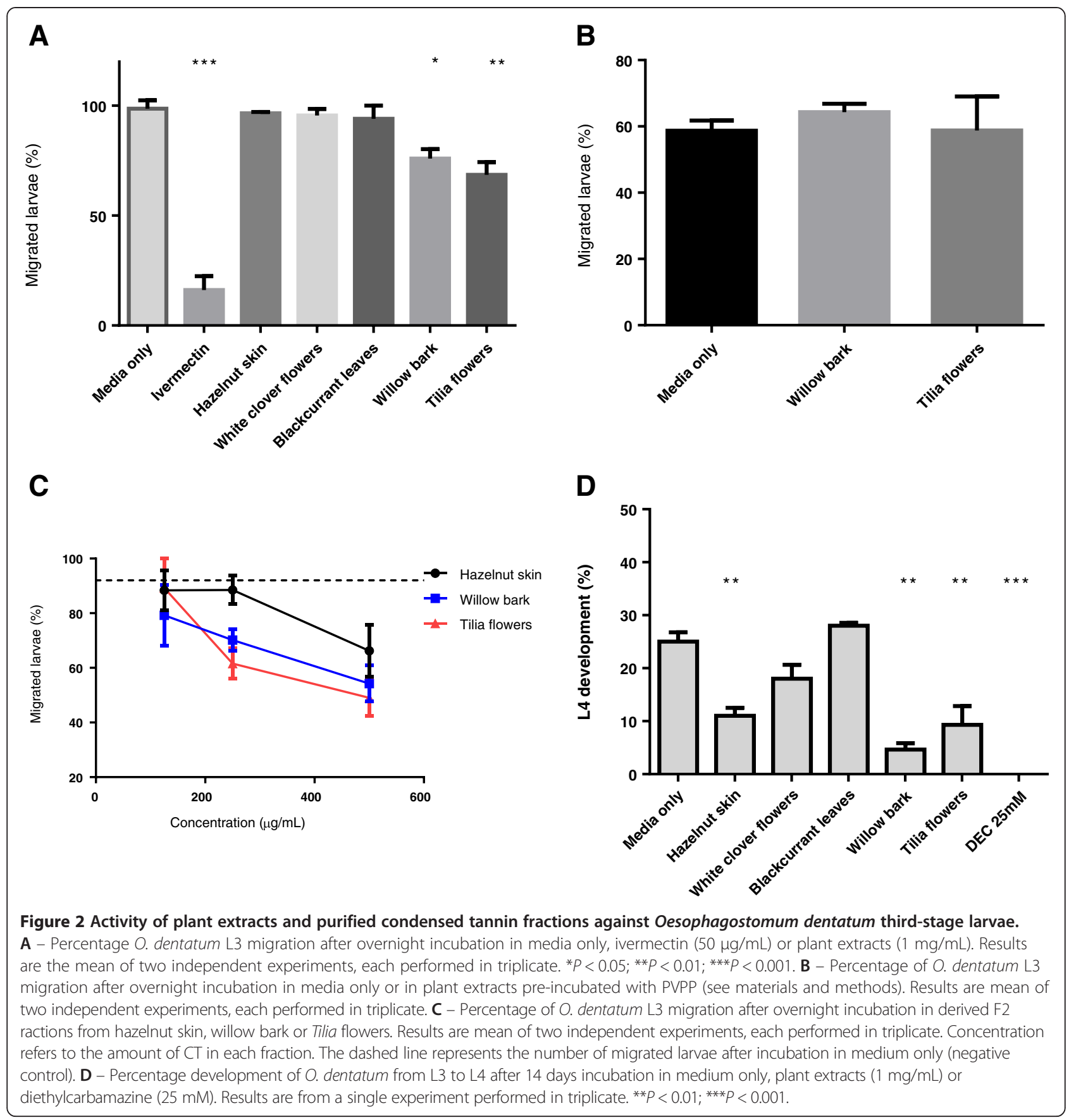

alone, $25 \%$ of L3 had moulted to the L4 stage. In contrast, the number of L3 that successfully moulted to L4 was significantly reduced after incubation in extract from hazelnut skins, willow bark or Tilia flowers (Figure 2D). No inhibition of moulting was observed after incubation with extracts from white clover flowers or blackcurrant. These data indicate that some $\mathrm{CTs}$ are able to interfere with the moulting process of O. dentatum.
Effects of extracts and tannin fractions on motility of fourth-stage larvae

Anthelmintic effects of the plant extracts and purified CT fractions were further assessed against in vitro cultured L4 parasites. After incubation in each of the five plant extracts at concentrations of 2 or $1 \mathrm{mg} / \mathrm{mL}$, a reduction in motility was noted in all parasites after 24 hours (Figure 3 ). In parasites exposed to extracts from white clover flowers, no further reductions in motility were observed over the 


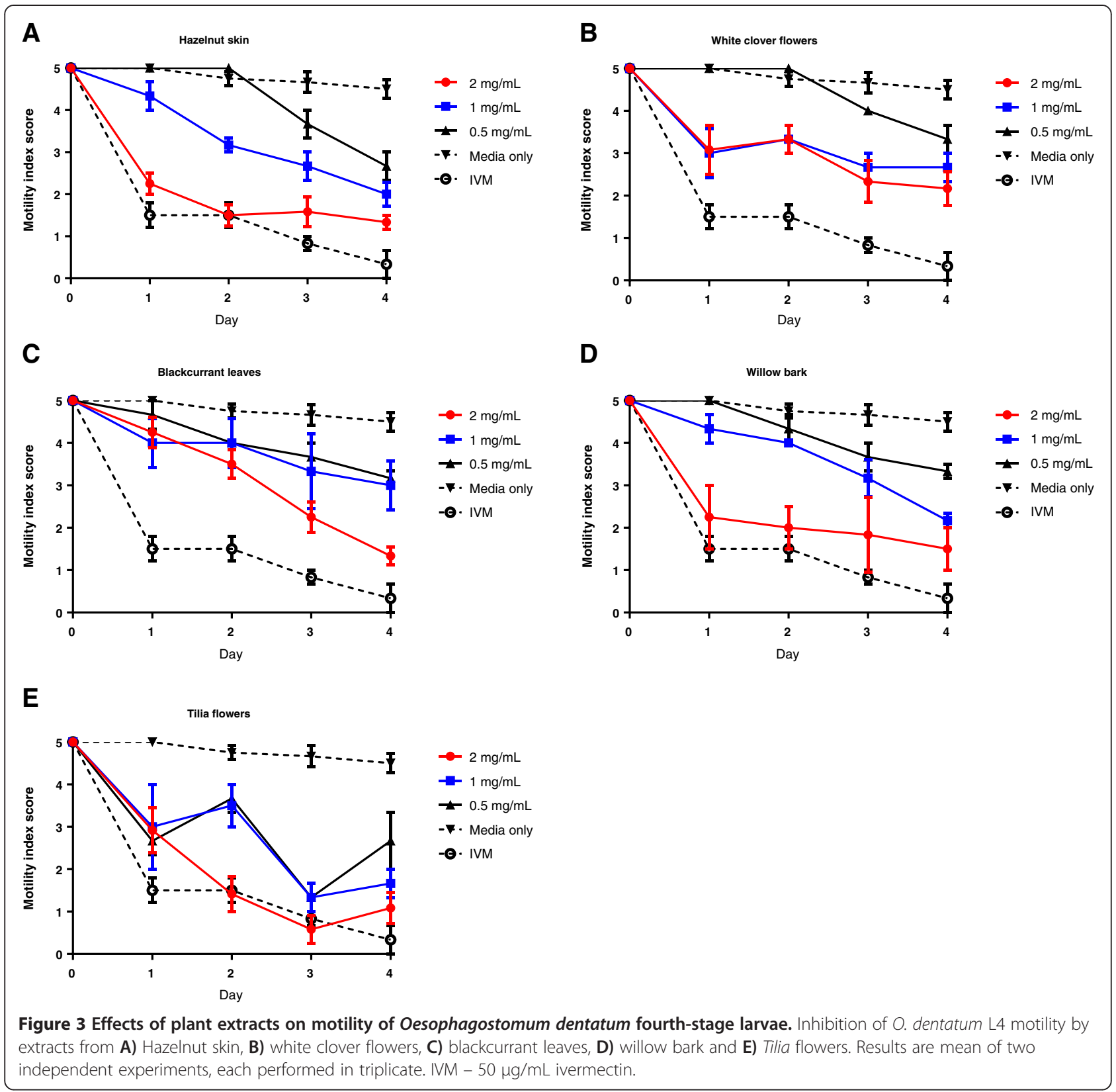

next 3 days (Figure 3B), whereas motility continued to decline in worms exposed to the other four extracts, resulting in a near-cessation of movement at the high concentrations of extract by the end of the experiment (Figure 3A, C-E).

To explore the relative contribution of the different $\mathrm{CT}$ molecules to the observed effects, isolated F2 fractions from all five extracts were then tested in the motility assay, as anthelmintic activity had been associated with this fraction in the L3 migration assay. The concentrations of CT were normalised between fractions to allow direct comparison between samples. Similar to the results with the acetone/water extracts, incubation in the F2 fractions resulted in reductions of motility after 24 hours of incubation (Figure 4). Motility continued to decline substantially in L4 exposed to fractions of Tilia flowers, resulting in near-paralysis by the end of the experiment (Figure 4E). In worms exposed to the other four fractions, further reductions in motility were less apparent, even after four days of incubation; however, motility did not return to preincubation levels (Figure 4A-D).

\section{Effects of hazelnut skin-extract on motility and morphology of adult worms}

We also assessed the ability of hazelnut extract to reduce the motility of adult worms recovered directly from pigs - 


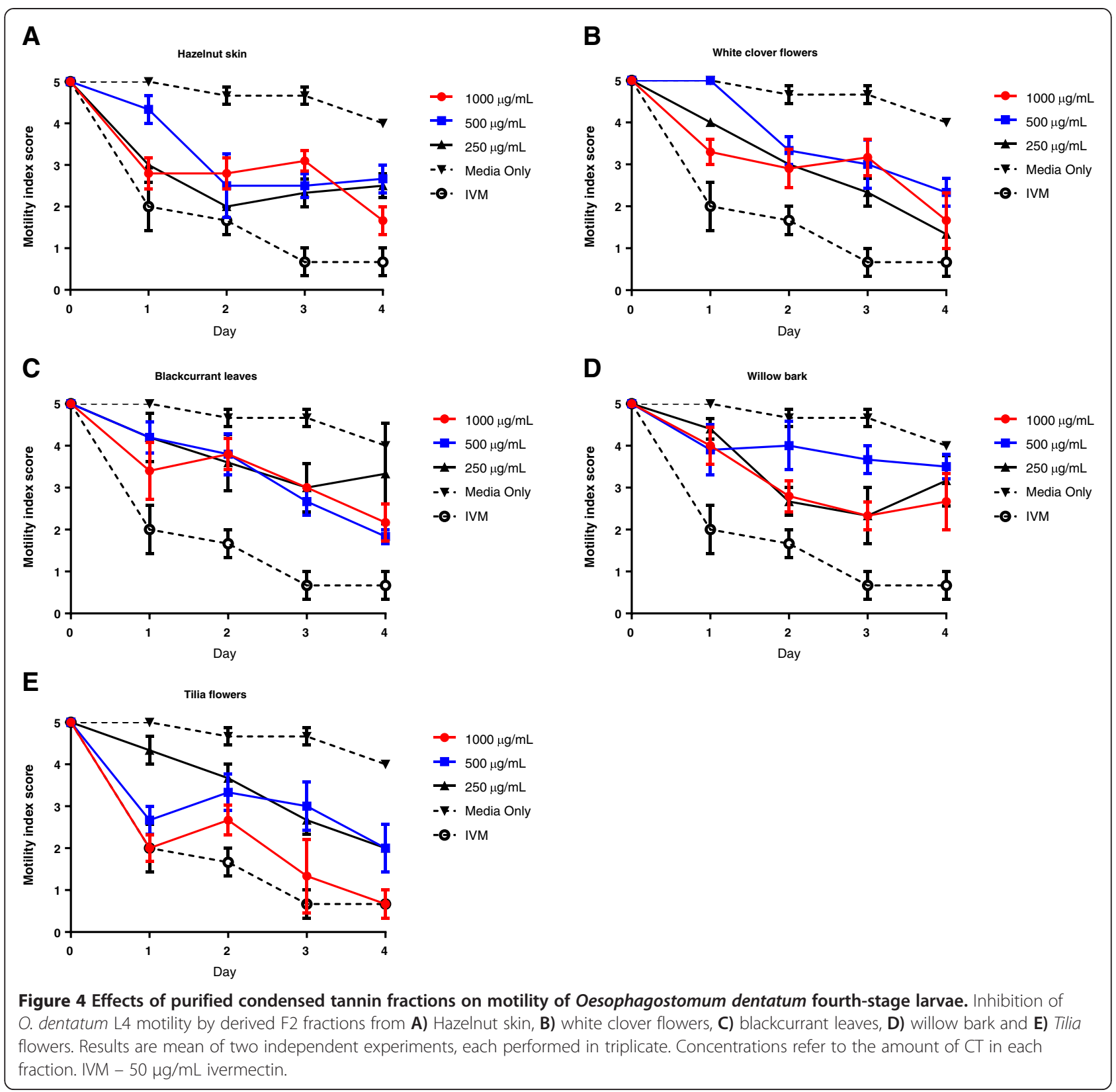

only a single extract was used here due to limited numbers of adult parasites. There was a clear dose-dependent reduction in motility, with worms exposed to $1 \mathrm{mg} / \mathrm{mL}$ of extract displaying little or no movement after 12 hours of incubation (Figure 5), and after 48 hours worms exposed to concentrations of $\geq 250 \mu \mathrm{g} / \mathrm{mL}$ showed clear signs of paralysis and structural damage including blebbing and a 'rough' cuticle. To explore further the structural damage caused by exposure to $\mathrm{CT}$, the cuticle of worms exposed to the highest concentration of CT $(1 \mathrm{mg} / \mathrm{mL})$ was examined by transmission electron microscopy, which revealed clear damage with marked irregularity of the cuticular surface, in contrast to the smooth surface observed in control worms (Figure 5). In addition, the underlying hypodermis appeared to be torn and detached from the basal layer of the cuticle, with rupturing and lesions also observed within the hypodermis itself (Figure 5).

\section{Discussion}

Continued reliance on mass drug administration with a limited number of synthetic anthelmintics has the potential to place heavy selection pressure on drug-resistant parasites, and widespread anthelmintic drug resistance is already a serious problem in many livestock production systems. The use of natural dietary compounds has the potential to be a complementary control option which 
A

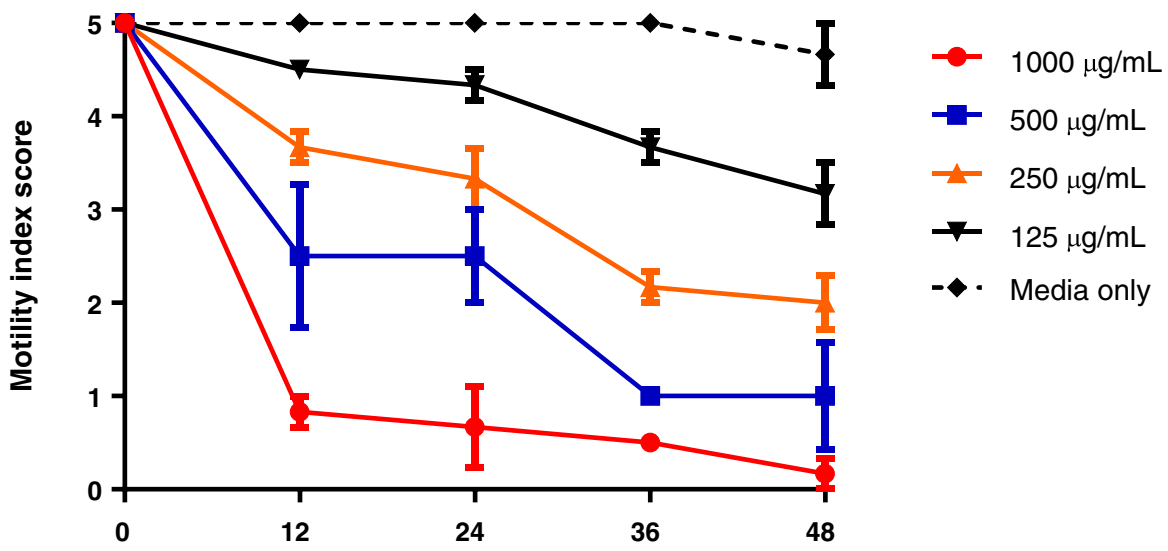

Time (hours)

B
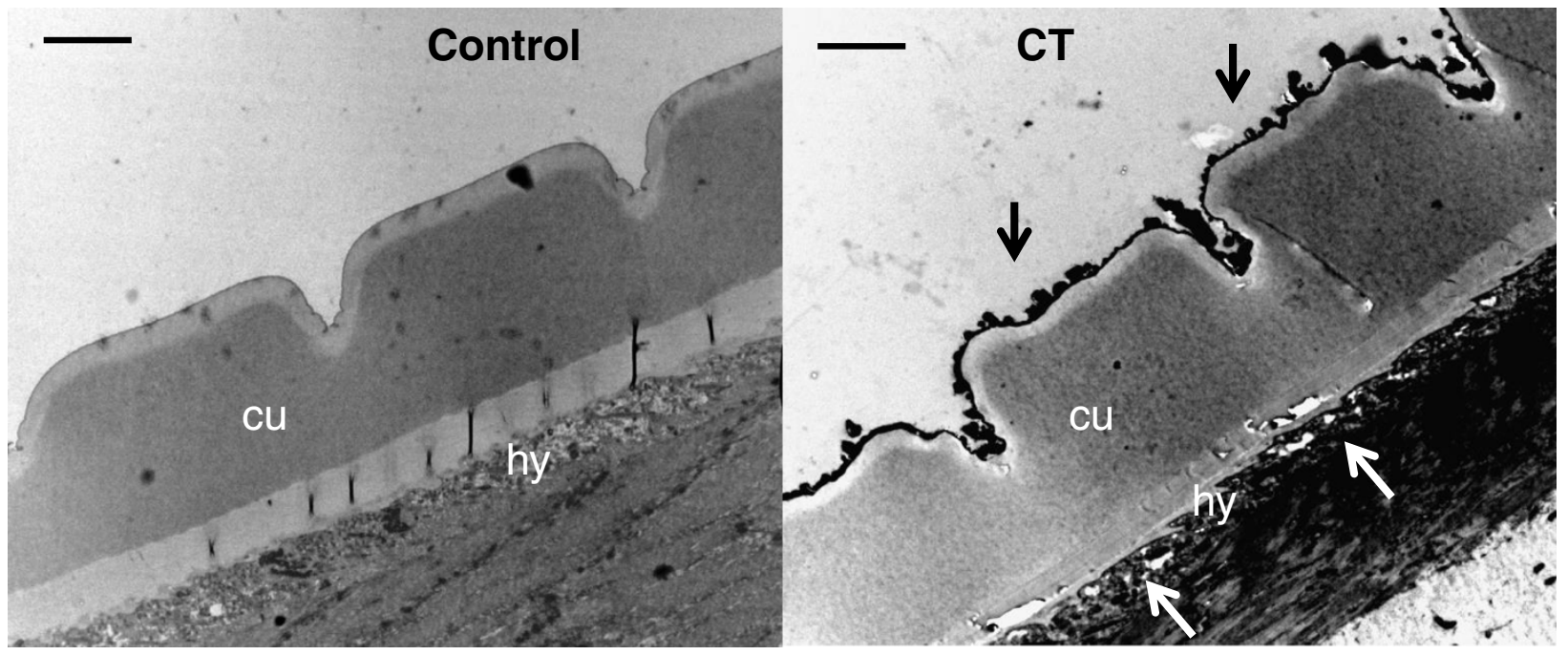

Figure 5 Effects of condensed tannins on motility and morphology of adult Oesophagostomum dentatum. A - Inhibition of $O$. dentatum adult motility by hazelnut skin extract. Results are from a single experiment performed in triplicate. B - Transmission-electron microscopy of thin sections of adult worms exposed to only medium ('control') or $1 \mathrm{mg} / \mathrm{mL}$ of hazelnut skin extract ('CT') for 24 hours. 'cu' - cuticle; 'hy' - hypodermis. Note the rough cuticle in worms exposed to CT (black arrows), and rupture and detachment of the hypodermis (white arrows). Scale bar =2 $\mu \mathrm{M}$.

may reduce this reliance on drug treatment, and slow the development of resistance. Here we have carried out a comprehensive in vitro assessment of the effects of CT from five different plant sources on one of the most prevalent parasites of pigs, O. dentatum.

We found that the development of free-living larvae was potently inhibited by all five extracts from CTcontaining plants. This larval development assay is commonly used to screen potential anthelmintic compounds [26], however it has the drawback of focusing on stages of the parasite life cycle which are not exposed to the compound in vivo. Therefore, it cannot be automatically assumed that results observed with these free-living stages can be extrapolated to the parasitic stages found within the GI tract. Indeed, we found that a concentration $(1 \mathrm{mg} / \mathrm{mL})$ that completely inhibited larval development with all five extracts had only modest inhibitory effects against migration of exsheathed L3 parasites. Hazelnut skin, blackcurrant leaf and white clover flower extracts had no significant effects on larval migration, whilst extracts from willow bark and Tilia flowers did significantly inhibit migration but only by $20-30 \%$ - an 
effect considerably less than the $95 \%$ inhibition observed after incubation in ivermectin. Therefore, it is apparent that whilst some CT-containing extracts can inhibit migration of infective larvae, overall the anthelmintic effect is less pronounced against this early parasitic stage than against free-living larvae. Therefore, our results highlight the importance of assessing putative anthelmintic compounds against not only free-living parasites but also parasitic stages. The active compounds within Tilia flowers and willow bark were confirmed as CT by both PVPP-incubation and fractionation experiments, which demonstrated that anthelmintic activity was retained in CT fractions of $80-90 \%$ purity. It is interesting to contrast the results obtained here with our previous work with another pig nematode, $A$. suum, where incubation of L3 in comparable concentrations of CT led to a substantially higher inhibition of larval migration [17]. This suggests that not all helminth species are equally susceptible to the anthelmintic effects of $\mathrm{CT}$, and careful assessment of activity is necessary before the suitability of these natural anti-parasitic compounds can be considered as viable control options in varying host-parasite systems.

Moulting of L3 to L4 parasites was significantly reduced after incubation in hazelnut skin, willow bark or Tilia flower extracts. Moreover, it was apparent that the motility of L4 was also reduced after incubation in extracts and fractions from the five plant sources. In the L3/L4 moulting assay, the motility and survival of the larvae was not affected by CT (data not shown), indicating that the reduction of development to $\mathrm{L} 4$ may be related to inhibition of specific metabolic processes involved in the moulting process [27]. To our knowledge, this is the first report that plant extracts can specifically inhibit the moulting process of a parasitic nematode, and further experiments are warranted to determine the mechanisms behind this inhibitory effect. It is also notable that adult worms appeared to be quite susceptible to the anthelmintic properties of CT. Further experiments with adult worms were not possible due to lack of parasite material, however the clear and rapid reductions in motility in adults exposed to the hazelnut skin extract indicate a potent effect. This suggests that dietary CT could potentially be used as a therapeutic option to specifically target adult worms and potentially prevent egg excretion and environmental contamination; however, further experiments will be necessary to explore this possibility. Overall, these experiments highlighted that while all stages of the parasite life cycle appeared to have some susceptibility to the plant extracts, clear differences between stages were observed. Interestingly, a recent study has also reported that a selection of medicinal plant extracts have increased activity towards Caenorhabditis elegans adults as compared to L3 or L4 stages, perhaps consistent with our results [28]. This has clear implications for the potential use of these plants for parasite control measures, as well as raising mechanistic questions about the observed anthelmintic activities.

Besides the quantity of CT, the structural characteristics of the polymers such as molecular weight and the ratio of different flavanol monomer units are known to affect anthelmintic activity $[29,30]$. In the current experiments, anthelmintic activity appeared to be associated with the higher molecular weight F2 fractions, suggesting that the anthelmintic activity in the extracts derives from CT with a high degree of polymerisation, consistent with previous studies with $A$. suum and O. ostertagi $[17,29]$. Moreover, extracts from Tilia flowers and willow bark were more potent in the L3 migration assays than hazelnut skin, white clover flower and blackcurrant extracts. Furthermore, Tilia flower, willow bark and hazelnut skin extracts significantly inhibited larval moulting, whereas white clover flower and blackcurrant extracts had no effect. Extracts and fractions derived from Tilia flowers also appeared to be most potent in the L4 motility assays, even when purified fractions were used with normalised concentrations of CT. Therefore, the observed differences in potency between the different acetone/water extracts do not appear to be related to the quantity of CT. Tannins in Tilia flowers, hazelnut skin and willow bark are comprised mainly of PC, whilst white clover flowers and blackcurrant leaves contain PD $[17,31,32]$. This suggests that PC-rich extracts/fractions may have higher activity towards $O$. dentatum, which is somewhat surprising given that PD are generally considered to have higher biological activity than PC (and the same applies to their corresponding, monomeric flavanol constituents) due to an extra hydroxyl group in the Bring favouring increased hydrogen bonding with proteins [33]. Indeed, the monomeric constituents of PD (gallocatechin and epigallocatechin) have been shown to have higher anthelmintic activity than catechin and epicatechin against the ruminant nematodes Trichostrongylus colubriformis and Haemonchus contortus [30,34], and a correlation has also been noted between the proportion of $\mathrm{PD}$ in polymeric $\mathrm{CT}$ and anthelmintic activity against O. ostertagi [29]. Further studies with larger panels of well-characterised CT fractions will be necessary to determine if the PC:PD ratio plays a role in the anthelmintic effects observed here.

Given the overall lower efficacy of the isolated molecules against $O$. dentatum than comparable studies with A. suum [17], it may be that the mechanism of action of $\mathrm{CT}$ against $O$. dentatum is subtly different than to other helminths. At present, the anthelmintic mode-of-action of CT is not known, but is proposed to involve biochemical interactions between $\mathrm{CT}$ and proline-rich proteins on the nematode sheath or cuticle that interfere with both worm motility and feeding, and also key metabolic 
processes such as exsheathment (and perhaps also moulting, as suggested by our current data). This is supported by electron microscopy studies of worms exposed to $\mathrm{CT}$ that demonstrate direct structural damage to the cuticle $[17,35]$, consistent with the ultrastructural changes we observed in the present study with adult O. dentatum. Such a mechanism would appear to be fairly non-specific and broad-spectrum in nature, hence it is interesting to note the differences in susceptibility between different nematodes. O. dentatum falls within the Strongyloididea superfamily of nematodes, a distinct family from the Ascaraidoidea (e.g. A. suum) and Trichostrongylidae (e.g. $T$. colubriformis and O. ostertagi) superfamilies [36], and this divergence may represent biological differences that determine susceptibility to $\mathrm{CT}$. Comparative studies of these two nematodes, including transcriptomic and proteomic analyses of worms exposed to equivalent amounts of CT, may shed some light on the more precise mechanisms of the anthelmintic effect, and such studies are on-going in our laboratory. The marked differences in the response of $O$. dentatum during free-living development and adults to CT, compared to L3 stages, is also deserving of further studies to determine the mechanisms responsible.

An important consideration is how these in vitro results may be translated to in vivo studies in pigs. Experimental use of CT as an alternative anthelmintic in livestock has been characterised by two (somewhat overlapping) approaches, these being either short term consumption with the aim of either reducing establishment of incoming larvae or targeted therapeutic administration to remove adult worms $[37,38]$, or long-term incorporation into the diet with the aim of disturbing key processes throughout the parasite life cycle, resulting in cumulative anthelmintic effects $[39,40]$. The fact that multiple processes in the life cycle of $O$. dentatum are affected by $\mathrm{CT}$ raises the possibility of a cumulative anthelmintic effect in vivo, whereby if a diet is consumed which contains CT, the additive sub-lethal effects against the different life cycle stages may reduce the viability and perhaps fecundity of worms as they mature within the host. In addition, the apparent susceptibility of adult worms to relatively low concentrations of $\mathrm{CT}$ raises the possibility of short-term feeding of $\mathrm{CT}$ as a complementary or alternative option to therapeutic drug treatment. Further in vivo studies to explore these options are necessary.

Moreover, the location of $O$. dentatum within the GI tract needs to be considered. Whilst there are a plethora of in vivo studies on the effects of CT-containing plants on worm infections in sheep and goats, as well as several rodent studies, most of these have focused on worms that reside in the stomach or small intestine, and thus there is only limited information on the effects of dietary CT upon large intestinal parasites. There is some evidence that pigs fed tannin-containing acorns (albeit hydrolysable tannins, quite different in structure to the CT tested here) have marked reductions in $O$. dentatum egg excretion [41]. However, sheep grazing the PC-rich forage Lotus corniculatus did not have reductions in burdens of Oesophagostomum spp., despite significant reductions in numbers of the abomasal parasite $H$. contortus and the small intestinal worm Cooperia curticei [42]. These authors speculated that changes to the structure of $\mathrm{CT}$ molecules in distal parts of the GI tract could affect anthelmintic activity. Whilst polymeric CT molecules are poorly absorbed from the GI tract [43], some fermentation and/or bacterial degradation of $\mathrm{CT}$ can occur in the monogastric large intestine [44-46]. This implies that dietary CT that are efficacious against parasites residing in the upper regions of the GI tract may not have comparable activity against parasites in distal regions, due to increased breakdown and a decrease in polymerization which may reduce potency. Therefore, further studies should focus on elucidating concentrations of $\mathrm{CT}$ in the local gut environments and whether polymeric CT retain their structure and anthelmintic activity through the entirety of the GI system.

\section{Conclusion}

We have for the first time shown anthelmintic effects of CT-containing plant extracts and purified CT fractions against $O$. dentatum, and demonstrated that free-living/ non-infective stages and adults appear to be highly susceptible to the effects of CT, whereas L4 are less susceptible and L3 are only modestly affected. Moreover, the moulting of L3 to L4 can be inhibited by CT, suggesting that specific, key processes in the parasite life cycle can be disrupted by CT. These data encourage further investigations to determine in vivo efficacy in pigs. In addition, further mechanistic studies, such as the relationship between the fine structure of CT molecules and anthelmintic activity, are also a high priority.

Abbreviations
CT: Condensed tannins; PC: Procyanidins; PD: Prodelphinidins.

Competing interests

The authors declare that they have no competing interests.

\section{Authors' contributions}

ARW, IMH and SMT conceived and designed the study. ARW and OD performed the parasitology experiments. HMR and CF prepared and analysed plant extracts and fractions. ARW analysed the experimental data and wrote the paper. All authors read and approved the final manuscript.

\section{Acknowledgements}

The authors are grateful to Lotte Christiansen and Tina Alstrup Hansen for assistance, Hervé Hoste (INRA, Toulouse) for providing the hazelnut skin material, and the Core Facility for Integrated Microscopy, University of Copenhagen. This work was funded by the Danish Council for Independent Research (Technology and Production Sciences, Grant \# 12-126630) and through the EU (Marie Curie ITN 'LegumePlus', PITN-GA-2011-289377). 


\section{Author details}

${ }^{1}$ Department of Veterinary Disease Biology, Faculty of Health and Medical Sciences, University of Copenhagen, Frederiksberg, Denmark. ${ }^{2}$ Chemistry and Biochemistry Laboratory, School of Agriculture, Policy and Development, University of Reading, Reading, UK

Received: 9 September 2014 Accepted: 3 November 2014 (1.

\section{References}

1. Keiser J, Utzinger J: Efficacy of current drugs against soil-transmitted helminth infections: Systematic review and meta-analysis. JAMA 2008, 299(16):1937-1948.

2. Charlier J, van der Voort M, Kenyon F, Skuce P, Vercruysse J: Chasing helminths and their economic impact on farmed ruminants. Trends Parasitol 2014, 30(7):361-367.

3. Fitzpatrick JL: Global food security: The impact of veterinary parasites and parasitologists. Vet Parasitol 2013, 195(3-4):233-248.

4. Sutherland IA, Leathwick DM: Anthelmintic resistance in nematode parasites of cattle: a global issue? Trends Parasitol 2011, 27(4):176-181.

5. Sargison ND: Pharmaceutical treatments of gastrointestinal nematode infections of sheep-Future of anthelmintic drugs. Vet Parasitol 2012, 189(1):79-84.

6. Githiori JB, Höglund J, Waller PJ: Ethnoveterinary plant preparations as livestock dewormers: practices, popular beliefs, pitfalls and prospects for the future. Anim Health Res Rev 2005, 6(01):91-103.

7. Athanasiadou S, Githiori J, Kyriazakis I: Medicinal plants for helminth parasite control: facts and fiction. Animal 2007, 1(09):1392-1400.

8. Tolossa K, Debela E, Athanasiadou S, Tolera A, Ganga G, Houdijk J: Ethnomedicinal study of plants used for treatment of human and livestock ailments by traditional healers in South Omo, Southern Ethiopia. J Ethnobiol Ethnomed 2013, 9(1):32.

9. Cowan MM: Plant Products as Antimicrobial Agents. Clin Microbiol Rev 1999, 12(4):564-582.

10. Mueller-Harvey I: Unravelling the conundrum of tannins in animal nutrition and health. J Sci Food Agric 2006, 86(13):2010-2037.

11. Martinez-Micaelo N, González-Abuín N, Ardèvol A, Pinent M, Blay MT: Procyanidins and inflammation: Molecular targets and health implications. Biofactors 2012, 38(4):257-265.

12. González R, Ballester I, López-Posadas R, Suárez MD, Zarzuelo A, MartínezAugustin O, Medina FSD: Effects of flavonoids and other polyphenols on inflammation. Crit Rev Food Sci Nutr 2011, 51(4):331-362.

13. Butter NL, Dawson JM, Wakelin D, Buttery PJ: Effect of dietary condensed tannins on gastrointestinal nematodes. J Agric Sci 2001, 137(04):461-469.

14. Heckendorn F, Häring DA, Maurer V, Zinsstag J, Langhans W, Hertzberg H: Effect of sainfoin (Onobrychis viciifolia) silage and hay on established populations of Haemonchus contortus and Cooperia curticei in lambs. Vet Parasitol 2006, 142(3-4):293-300.

15. Brunet $\mathrm{S}$, Jackson F, Hoste H: Effects of sainfoin (Onobrychis viciifolia) extract and monomers of condensed tannins on the association of abomasal nematode larvae with fundic explants. Int J Parasito/ 2008, 38(7):783-790.

16. Novobilský A, Mueller-Harvey I, Thamsborg SM: Condensed tannins act against cattle nematodes. Vet Parasitol 2011, 182(2-4):213-220.

17. Williams AR, Fryganas C, Ramsay A, Mueller-Harvey I, Thamsborg SM: Direct anthelmintic effects of condensed tannins from diverse plant sources against Ascaris suum. PLoS One 2014, 9(5):e97053.

18. Roepstorff A, Mejer H, Nejsum P, Thamsborg SM: Helminth parasites in pigs: New challenges in pig production and current research highlights. Vet Parasitol 2011, 180(1-2):72-81.

19. Hale OM, Stewart TB, Marti OG, Wheat BE, McCormick WC: Influence of an experimental infection of nodular worms (Oesophagostomum spp.) on performance of pigs. J Anim Sci 1981, 52(2):316-322.

20. Stewart TB, Hale OM: Losses to internal parasites in swine production. J Anim Sci 1988, 66(6):1548-1554.

21. Gerwert S, Failing K, Bauer C: Prevalence of levamisole and benzimidazole resistance in Oesophagostomum populations of pig-breeding farms in North Rhine-Westphalia, Germany. Parasitol Res 2002, 88(1):63-68.

22. Dangolla A, Bjørn H, Willeberg P, Barnes EH: Faecal egg count reduction percentage calculations to detect anthelmintic resistance in Oesophagostomum spp. in pigs. Vet Parasitol 1997, 68(1-2):127-142.
23. Gasser RB, Cottee P, Nisbet AJ, Ruttkowski B, Ranganathan S, Joachim A: Oesophagostomum dentatum - Potential as a model for genomic studies of strongylid nematodes, with biotechnological prospects. Biotechnol Adv 2007, 25(3):281-293.

24. Várady M, Bjørn H, Craven J, Nansen P: In vitro characterization of lines of Oesophagostomum dentatum selected or not selected for resistance to pyrantel, levamisole and ivermectin. Int J Parasitol 1997, 27(1):77-81.

25. Stepek G, Buttle DJ, Duce IR, Lowe A, Behnke JM: Assessment of the anthelmintic effect of natural plant cysteine proteinases against the gastrointestinal nematode, Heligmosomoides polygyrus, in vitro. Parasitology 2005, 130(02):203-211.

26. Kotze AC: Target-based and whole-worm screening approaches to anthelmintic discovery. Vet Parasitol 2012, 186(1-2):118-123.

27. Ondrovics M, Silbermayr K, Mitreva M, Young ND, Razzazi-Fazeli E, Gasser RB, Joachim A: Proteomic Analysis of Oesophagostomum dentatum (Nematoda) during Larval Transition, and the Effects of Hydrolase Inhibitors on Development. PLoS One 2013, 8(5):e63955.

28. Kumarasingha R, Palombo EA, Bhave M, Yeo TC, Lim DSL, Tu CL, Shaw JM, Boag PR: Enhancing a search for traditional medicinal plants with anthelmintic action by using wild type and stress reporter Caenorhabditis elegans strains as screening tools. Int J Parasitol 2014, 44(5):291-298.

29. Novobilský A, Stringano E, Hayot Carbonero C, Smith LMJ, Enemark HL, Mueller-Harvey I, Thamsborg SM: In vitro effects of extracts and purified tannins of sainfoin (Onobrychis viciifolia) against two cattle nematodes. Vet Parasitol 2013, 196(3-4):532-537.

30. Brunet S, Hoste H: Monomers of Condensed Tannins Affect the Larval Exsheathment of Parasitic Nematodes of Ruminants. J Agric Food Chem 2006, 54(20):7481-7487.

31. Esatbeyoglu T, Wray $V$, Winterhalter $P$ : Dimeric procyanidins: screening for B1 to B8 and semisynthetic preparation of B3, B4, B6, and B8 from a polymeric procyanidin fraction of White Willow bark (Salix alba). J Agric Food Chem 2010, 58(13):7820-7830.

32. Ropiak HM, Ramsay A, Mueller-Harvey I: Condensed tannins from European medicinal plants. In Polyphenol Communications (Proceedings of the XXVIIth International Conference on Polyphenols \& 8th tannin conference). Nagoya, Japan: Groupe Polyphenols; 2014:609-610.

33. Scalbert A: Antimicrobial properties of tannins. Phytochemistry 1991, 30(12):3875-3883.

34. Molan AL, Meagher LP, Spencer PA, Sivakumaran S: Effect of flavan-3-ols on in vitro egg hatching, larval development and viability of infective larvae of Trichostrongylus colubriformis. Int J Parasitol 2003, 33(14):1691-1698.

35. Brunet S, Fourquaux I, Hoste H: Ultrastructural changes in the third-stage, infective larvae of ruminant nematodes treated with sainfoin (Onobrychis viciifolia) extract. Parasitol Int 2011, 60(4):419-424.

36. Roberts LS, Janovy J: Foundations of Parasitology. 6th edition. Singapore: McGraw-Hill; 2000.

37. Athanasiadou S, Kyriazakis I, Jackson F, Coop RL: Direct anthelmintic effects of condensed tannins towards different gastrointestinal nematodes of sheep: in vitro and in vivo studies. Vet Parasitol 2001, 99(3):205-219.

38. Brunet S, Montellano CM-O, Torres-Acosta JFJ, Sandoval-Castro CA, AguilarCaballero AJ, Capetillo-Leal C, Hoste H: Effect of the consumption of Lysiloma latisiliquum on the larval establishment of gastrointestinal nematodes in goats. Vet Parasitol 2008, 157(1-2):81-88.

39. Hoste H, Martinez-Ortiz-De-Montellano C, Manolaraki F, Brunet S, OjedaRobertos N, Fourquaux I, Torres-Acosta JFJ, Sandoval-Castro CA: Direct and indirect effects of bioactive tannin-rich tropical and temperate legumes against nematode infections. Vet Parasitol 2012, 186(1-2):18-27.

40. Niezen JH, Robertson HA, Waghorn GC, Charleston WAG: Production, faecal egg counts and worm burdens of ewe lambs which grazed six contrasting forages. Vet Parasitol 1998, 80(1):15-27.

41. Salajpal K, Karolyi D, Beck R, Kis G, Vickovic I, Dikic M, Kovacic D: Effect of acorn (Quercus robur) intake on faecal egg count in outdoor reared black Slavonian pig. Acta Agric Slovenica 2004, 84(Supplement 1):173-178,

42. Ramírez-Restrepo CA, Barry TN, Pomroy WE, López-Villalobos N, McNabb WC, Kemp PD: Use of Lotus corniculatus containing condensed tannins to increase summer lamb growth under commercial dryland farming conditions with minimal anthelmintic drench input. Anim Feed Sci Tech 2005, 122(3-4):197-217.

43. Reed JD: Nutritional toxicology of tannins and related polyphenols in forage legumes. J Anim Sci 1995, 73(5):1516-1528. 
44. Déprez S, Brezillon C, Rabot S, Philippe C, Mila I, Lapierre C, Scalbert A: Polymeric proanthocyanidins are catabolized by human colonic microflora into low-molecular-weight phenolic acids. J Nutr 2000, 130(11):2733-2738.

45. Gu L, House SE, Rooney L, Prior RL: Sorghum bran in the diet dose dependently increased the excretion of catechins and microbial-derived phenolic acids in female rats. J Agric Food Chem 2007, 55(13):5326-5334.

46. Touriño S, Pérez-Jiménez J, Mateos-Martín ML, Fuguet E, Vinardell MP,

Cascante M, Torres $\mathrm{J}$ : Metabolites in contact with the rat digestive tract after ingestion of a phenolic-rich dietary fiber matrix. J Agric Food Chem 2011, 59(11):5955-5963.

doi:10.1186/s13071-014-0518-2

Cite this article as: Williams et al:: Assessment of the anthelmintic activity of medicinal plant extracts and purified condensed tannins against free-living and parasitic stages of Oesophagostomum dentatum. Parasites \& Vectors 2014 7:518.

\section{Submit your next manuscript to BioMed Central and take full advantage of:}

- Convenient online submission

- Thorough peer review

- No space constraints or color figure charges

- Immediate publication on acceptance

- Inclusion in PubMed, CAS, Scopus and Google Scholar

- Research which is freely available for redistribution 